\title{
Modeling and Simulation of Transmural Cellular Electromechanical Properties in Heart Failure
}

\author{
Y Zhang, GF Shou, R Huang, L Xia \\ Department of Biomedical Engineering, Zhejiang University, Hangzhou, China
}

\begin{abstract}
In this paper, we present a simulation study of transmural cellular electromechanical properties in heart failure based on a integrated and refined heart cell model of Luo-Rudy II model and modified Hunter-McCullochter Keurs mechanical model. The results show that prolonged action potential duration (APD) and APD rate-adaptation are more obviously transmurally heterogeneous in heart failure than those in control hearts. The results suggest that the differences of the electrical responses between failing cells and normal cells can cause slowing relaxation of the Ca2+ transient, and the difference of the Ca2+-TnC concentrations between fast and slow myocytes in failing hearts is smaller than in nonfailing hearts. It results in a decrease of force, which might diminish the role of mechanoelectric feedback, and then induce an increase of transmural APD gradient that might cause arrhythmia in heart failure. These results are in good accordance with experimental findings reported in the literatures and this electromechanical cardiac cell model might be very useful for further modeling and simulation of heart failure at both the tissue and the whole organ levels.
\end{abstract}

\section{Introduction}

Heart failure (HF) has emerged as one of the largest threats to the public health in the world. The relationship between alterations of repolarization and arrhythmia mechanisms in HF remains largely unknown [1]. And effects of heart failure on the mechanical function of the heart are also difficult to assess experimentally.

In the past decades, many electrical and mechanical heart models have been developed and used to investigate cardiac properties. However, the electromechanical mechanism of heart failure is not exactly clear so far. In this paper, we present a simulation study of transmural cellular electromechanical properties in heart failure based on an integrated and refined heart cell model.

\section{Methods}

\subsection{Cellular electrophysiology modeling}

In this study, the modified Luo-Rudy II model [2] is used to simulate action potential duration (APD) of fast contracting hearts (such as small rodents), whereas modified ten Tusscher et al. model [3] is used to simulate APD of slower contracting myocardium (such as cat, rabbit, dog). Compared to the slow contracting myocardium (Tusscher et al. model), the action potential in the faster myocardium (Luo-Rudy II model) has a higher amplitude and shorter duration. Based on experimental studies of electrophysiological heterogeneities in failing heart both in fast and slow myocytes, we modify the previous ventricular cell models.

Recent experiments have indicated that peak $I_{N a}$ and $I_{k l}$ density significantly decrease by $57 \%$ and $25 \%$ in human heart failure respectively [4]. There is also a reduction in the current density of $I_{t o l}$ and the reduction is different in the variable myocyte layers [3,5]. According to the results of experiments by Li et al. [6], the decrease of $I_{K s}$ in all three layers becomes more homogeneous in HF than that in nonfailing (NF) without a significant change in $I_{K r}$ [6]. The most common change of $I_{C a L}$ is a significant slowing of the whole-cell current decay [7], while a significantly enhancement of the peak average current of $I_{C a L}$ in HF has been reported [8]. Studies also show that the altered $\mathrm{Ca}^{2+}$ transients of the HF human myocytes are largely dependent on reduced sarcoplasmic reticulum (SR) $\mathrm{Ca}^{2+}$ uptake, storage, and release [9]. So, a reduction of the buffered calcium in the SR is incorporated into the HF model. Other currents such as $I_{N a K}, I_{b C a}$ and $I_{b N a}$ are changed in the same way to Priebe-Beuckelmann (PB) model [10].

\subsection{Modeling of mechanical properties of ventricular cells}

The mechanical cell model is constructed based on Hunter-McCulloch-ter Keurs (HMT) model [11], which used a curve fitting equation to describe the intracellular calcium concentration $\left[\mathrm{Ca}^{2+}\right]_{\mathrm{i}}$ :

$\mathrm{Ca}_{\mathrm{i}}(\mathrm{t})=\mathrm{Ca}_{0}+\left(\mathrm{Ca}_{\max }-\mathrm{Ca}_{0}\right) \frac{\mathrm{t}}{\tau_{\mathrm{Ca}}} \mathrm{e}^{1-t / \tau_{\mathrm{Ca}}}$
$\frac{\mathrm{dCa}_{\mathrm{TnC}}}{\mathrm{dt}}=\rho_{0} \mathrm{Ca}_{\mathrm{i}}\left(\mathrm{Ca}_{\mathrm{TnCmax}}-\mathrm{Ca}_{\mathrm{TnC}}\right)-\rho_{1}\left(1-\frac{\mathrm{T}}{\gamma \times \mathrm{T}_{0}}\right) \mathrm{Ca}_{\mathrm{TnC}}$

In this research, we replace (1) with $\left[\mathrm{Ca}^{2+}\right]_{\mathrm{i}}$ produced 
by simulation of highly developed models of cardiac cell membrane ion channels and transporters, in which the calcium induced-calcium release from SR is considered and provides the source of free myoplasmic calcium. HMT model is based on the experiment data of rat and ferret that belong to fast contracting myocytes. Mechanically fast muscle models are characterized by an increase in the rate constant for $\mathrm{Ca}^{2+}$ reuptake into the $\mathrm{SR}$ as well as a higher dissociation constant for the $\mathrm{Ca}^{2+}-\mathrm{TnC}$ complex [12]. As a result, we modify $\rho_{l}$ of (2) in order to simulate slow contracting myocytes.

\section{Results}

\subsection{Remodeling of heterogeneous AP and APD rate dependence in HF ventricular cells}

Based on the experimental studies of transmural electrophysiological heterogeneities in $\mathrm{HF}[6,13]$, cardiac AP characteristics (at $0.5-2 \mathrm{~Hz}$ ) are simulated in different ventricular cell types in control and in HF: epicardial, M and endocardial cells by changing the parameters setting (Fig.1 A). Fig.1 illustrates that HF causes transmural electrical remodeling and changes the transmural heterogeneity in APs, results to a reduction of transmural AP gradients which is same to some groups' findings [6]. But it is contrary to some recent findings [14]. Only in M cell, EADs can be observed when the pulses increase to 2 $\mathrm{Hz}$ in HF. It seems that $\mathrm{M}$ cells are more susceptible to EADs in failing hearts, as in response to cardioactive agents under nondiseased conditions.

We simulate the rate-dependence of APD in epicardial, midmyocardial, and endocardial layers of control and HF over a CL range from $4000 \mathrm{~ms}$ to $400 \mathrm{~ms}$. And we can find APDs of cells in all myocardial layers increased as CL was slowed. In control and HF, APD reached an apparent steady-state value at CL of $2000 \mathrm{~ms}$.

Most important for APD adaptation is $I_{\mathrm{CaL}}$ at fast rates [15], supported by the fact that eliminating $I_{C a L}$ from the model reduced adaptation (CL range of 8000 to $400 \mathrm{~ms}$ ) from 60 to $20 \mathrm{~ms}$ (66\% decrease). $I_{t o l}$ also plays a role: its elimination reduces adaptation by $25 \%$, which is decreased in repolarization may facilitates APD shortening at a fast rate.

\subsection{Selective enhancement of APD prolongation in $\mathrm{HF}$}

Recent study of Akar et al. [16] show APD prolongation in HF was markedly heterogeneous causing a significant rise (by 109\%) in transmural AP gradient They find $\mathrm{M}$ and endocardial cells underwent a more enhanced prolongation of their APD and significantly more enhanced sensitivity to rate compared with epicardial cells [14].

Based on the APD rate dependence analysis above, we simulated the AP shapes of different ventricular cell types in $\mathrm{HF}$ and the rate-dependence of APD again, with changing $I_{C a L}$ in epicardial cell (Fig.2). Results show the prolonged APD and APD rate-adaptation is markedly transmural heterogeneous (greater TDR), particularly in epicardial cells which is shorter and smaller than other cells, causing a significant rise in the transmural AP gradient similar to experiment data [16].
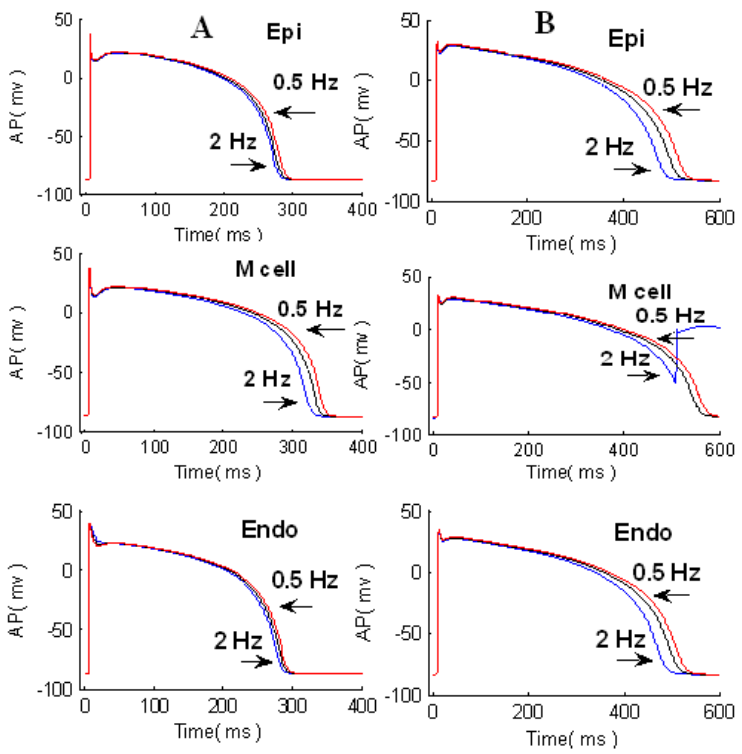

Fig.1. Simulated APs in cells from different regions of control and failing heart. A: AP in cells from the endocardium, midmyocardial layer ( $\mathrm{M}$ cell), and epicardium showed heterogeneity in morphology and AP duration (APD) at $0.5,1$ and $2 \mathrm{~Hz}$. B: APs simulated in cells form the Endo, $\mathrm{M}$ and epi layers of failing hearts.

\subsection{Simulation of $[\mathrm{Ca} 2+]$ i of heterogeneous myocytes in heart failure}

Based on experimental findings, compared to normal cells, the differences of the electrical responses in failing cells can cause reducing cardiac pump function due to the reducing of contractile force that may be the change of the intracellular $\mathrm{Ca}^{2+}$ transients $\left(\left[\mathrm{Ca}^{2+}\right]_{\mathrm{i}}\right)$ and $\mathrm{Ca}^{2+}-\mathrm{TnC}$ binding $\left(\left[\mathrm{Ca}^{2+}-\mathrm{TnC}\right]\right)$. We remodel the previous ventricular cell models with $\mathrm{Ca}$ handling by reducing SR $\mathrm{Ca}^{2+}$ uptake in the failing animal or human myocardium, which slows the decay of the $\mathrm{Ca}^{2+}$ transient and reduces $\mathrm{SR} \mathrm{Ca}^{2+}$ stores [9]. This leads to reduce $\mathrm{SR} \mathrm{Ca}^{2+}$ release, which induces additional $\mathrm{Ca}^{2+}$ influx during the plateau phase of the action potential, and further slow the decay of the $\mathrm{Ca}^{2+}$ transient. From Fig. 3A, C, model analyses demonstrate that the $\mathrm{Ca}^{2+}$ transient during the AP is markedly different in failing myocytes and NF ones, both for fast and slow contracting myocytes. So the concentrations of $\mathrm{Ca}^{2+}$ bound to the Ca-specific binding 
site on $\mathrm{TnC}$ changes are obviously different in $\mathrm{NF}$ and failing hearts (Fig. 3B, D). But, interestingly, we find that the difference of the $\mathrm{Ca}^{2+}-\mathrm{TnC}$ concentrations between fast and slow myocytes in failing hearts is much reduced than in NF hearts, which might cause a decrease of force difference in fast and slow myocytes in heart failure (Fig. 4). It corresponds well to experimental data [12].

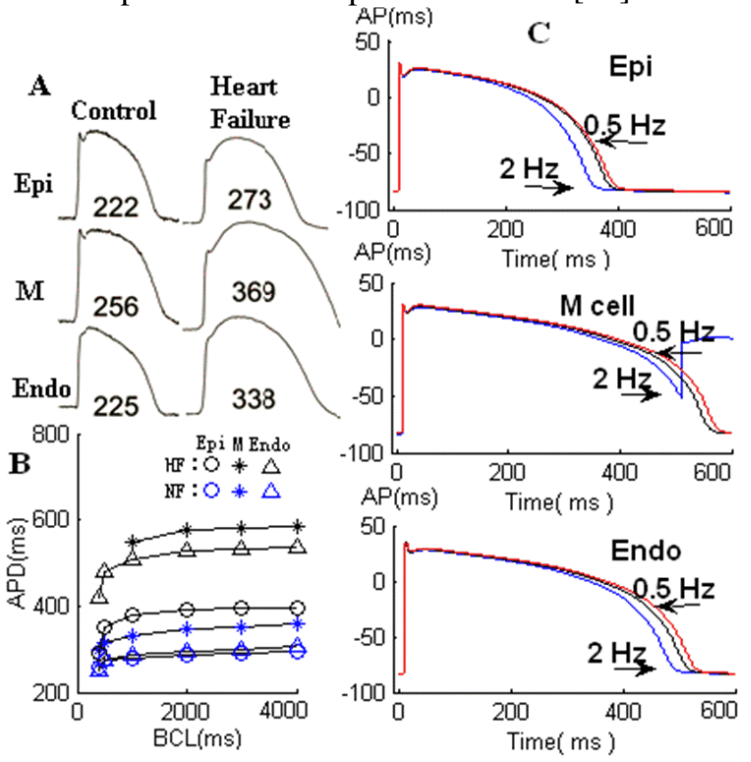

Fig.2. APD heterogeneities in failing myocardium. A: Representative APD recorded from the transmural of a control (left) and HF wedge (right). B: cycle length dependence of epicardial, M, and endo cardial APD in control and HF. C: APs simulated in cells from the endo, $\mathrm{M}$ and epi layers of failing hearts with changed parameter setting of current $I_{c a L}$ in Epi to explain enhancement transmural heterogeneities in HF.

\subsection{Ca2+ transient induced force decrease in $\mathbf{H F}$}

On the basis of the analysis of the changes of $\mathrm{Ca}$ handling in NF and failing hearts, we simulate the isometric force in fast and slow myocytes (Fig. 4). We find that the amplitude of the isometric force both in fast and slow myocytes is decreased, as many investigators reported [17]. And we also find that the fast myocytes reach peak force earlier and relax faster than the slow ones [12] (Fig. 4) in NF hearts. Both fast and slow myocytes in heart failure, velocity of contraction is reduced and magnitude of contraction is attenuated [18]. Thus, in terms of the classical force-velocity concept, in heterogeneous myocardium, power is reduced in heart failure since both force and velocity are reduced [18].

One consequence of coupling the mechanics to electrophysiology is that the binding of $\mathrm{Ca}^{2+}$ to $\mathrm{TnC}$ alters the $\mathrm{Ca}^{2+}$ transient that affects $\mathrm{Na}^{+}-\mathrm{Ca}^{2+}$ exchange and hence APD. Mechanically induced changes in APD in heterogeneous myocardium might explain the discrepancy of APD data of cells from different layers of the ventricular war: isometric sub-epicardial (fast) cells tend to have shorter APD than isometric sub-endocardial (slow) ones, however, mechanoelectric feedback (MEF) may contribute to a reduction in transmural APD gradients in the intact human heart [5]. But In heart failure, a change of intracellular $\mathrm{Ca}^{2+}$ transients $\left(\left[\mathrm{Ca}^{2+}\right]_{\mathrm{i}}\right)$ and $\mathrm{Ca}^{2+}-\mathrm{TnC}$ binding $\left(\left[\mathrm{Ca}^{2+}-\mathrm{TnC}\right]\right)$ results in a decrease of force, which might diminish the role of MEF, then induce an increase of transmural APD gradients. It may explain that the electrophysiological heterogeneities in heart failure might contribute to arrhythmogeneity [16].
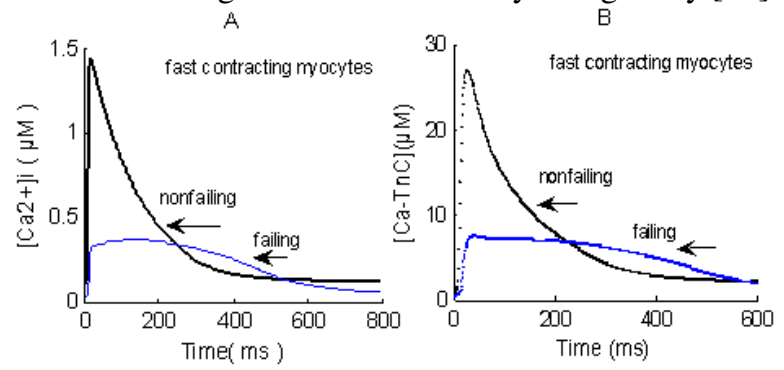

$\mathrm{C}$
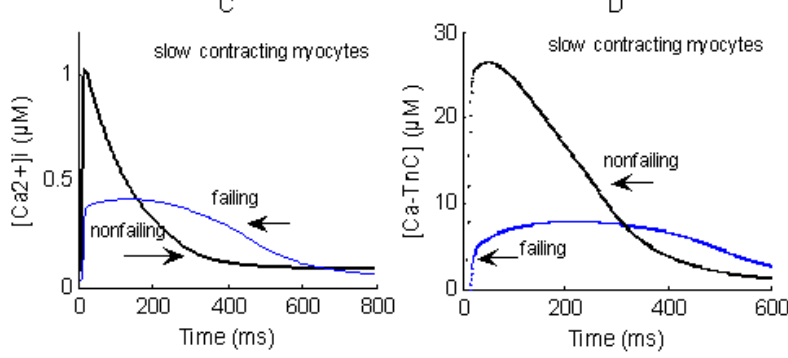

Fig.3. Simulation result of the intracellular $\left[\mathrm{Ca}^{2+}\right]_{\mathrm{i}}$ transient $\left(\left[\mathrm{Ca}^{2+}\right]_{\mathrm{i}}\right)$ and the concentration of $\mathrm{Ca}^{2+}$ bound to $\mathrm{TnC}$ in a nonfailing and failing myocyte at a stimulation frequency of $0.5 \mathrm{~Hz}$. (A), (B) fast myocytes. (C), (D) slow myocytes. (A), (C) the intracellular $\left[\mathrm{Ca}^{2+}\right]_{\mathrm{i}}$ transient $\left(\left[\mathrm{Ca}^{2+}\right]_{\mathrm{i}}\right)$. (B), (D) the concentration of $\mathrm{Ca}^{2+}$ bound to $\mathrm{TnC}$ due to $\left[\mathrm{Ca}^{2+}\right]_{\mathrm{i}}$ in $(\mathrm{A}),(\mathrm{C})$ respectively.
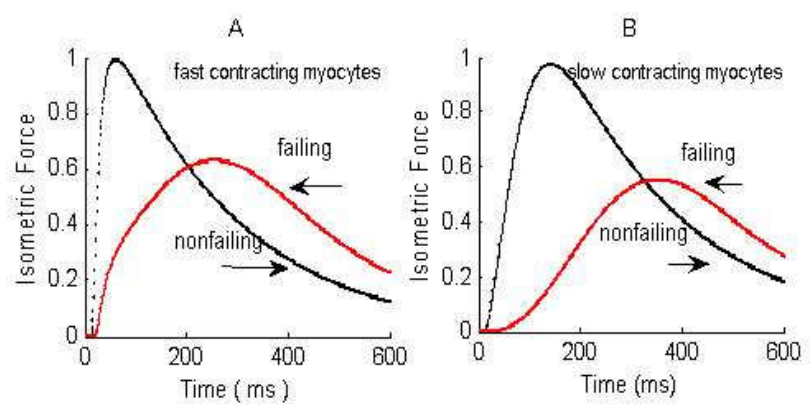

Fig.4. Contraction relaxation cycles in failing and nonfailing myocytes during isometric contractions. (A) fast myocytes. (B) slow myocytes. 


\section{Discussion and conclusions}

In this paper, we present a simulation study of transmural cellular electromechanical properties in heart failure based on an integrated and refined heart cell model. In the electrical property investigation, we simulate the action potential shapes of different ventricular cell types, and determine the changes of transmural heterogeneity of cellular electrophysiology in nonfailing and failing hearts. The results show that prolonged action potential duration (APD) and APD rateadaptation are more obviously transmural heterogeneous in heart failure than those in control heart, which may be due to enhanced transmural heterogeneities of $I_{C a L}$ and $I_{K s}$ in myocytes. In the mechanical property investigation, we study cellular mechanical properties of both fast and slow contracting myocytes in heart failure. The results suggest that the differences of the electrical responses between failing cells and normal cells can cause slowing relaxation of the $\mathrm{Ca}^{2+}$ transient, and the difference of the $\mathrm{Ca}^{2+}-\mathrm{TnC}$ concentrations between fast and slow myocytes in failing hearts is much reduced than in nonfailing hearts. It results in a decrease of force, which might diminish the role of MEF, then induce an increase of transmural APD gradients. It might cause arrhythmia in heart failure. These results are in good accordance with experimental findings reported in the literatures and this electromechanical cardiac cell model might be very useful for further modeling and simulation of heart failure at both the tissue and the whole organ levels.

However, the present model has several limitations. For example, in simulation of $\left[\mathrm{Ca}^{2+}\right]_{\mathrm{i}}$ transients, since the understanding of the exact mechanisms involved is incomplete, it is only an approximation of the complex nature of the intracellular $\mathrm{Ca}^{2+}$ homeostasis. Such problems should be further investigated in the future.

\section{Acknowledgements}

This project is supported by the 973 National Key Basic Research \& Development Program (2003CB716106) and National Natural Science Foundation of China (30370400).

\section{References}

[1] Rodan DM. A Surprising New Arrhythmia Mechanism in Heart Failure. Circ Res 2003; 93: 589-591.

[2] Luo CH and Rudy Y. A dynamic model of the cardiac ventricular action potential: I. Simulations of ionic currents and concentration changes. Circ Res 1994; 74: 1071-1096.

[3] ten Tusscher KHWJ, Noble D, Noble PJ and Panfilov AV. A model for human ventricular tissue. Am J Physiol Heart Circ Physiol 2004; 286: 1573-1589.

[4] Valdivia CR, et al. Increased late sodium current in myocytes from a canine heart failure model and from failing human heart. J Mol Cell Cardio 2005; 38: 475-483.

[5] Tarrart P, Sutton P, Opthof T, Coronel R and Kallis P. Electrotonic cancellation of transmural electrical gradients in the left ventricle in man. Prog Biophys Mol Biol 2003; 82: 243-254

[6] Li G, Lau CP, Ducharme A, Tardif JC and Nattel S. Transmural action potential and ionic current remodeling in ventricles of failing canine hearts. Am J Physiol Heart Circ Physiol 2002; 283: H1031-H1041.

[7] Tomaselli GF and Zipes DP. What causes sudden death in heart failure? Circ Res 2004; 95: 754-763

[8] Schröder F, et al. Increased availability and open probability of single L-type calcium channels from failing compared with nonfailing human ventricle. Circulation 1998; 98: 969-976.

[9] Piancentino VIII, et al. Cellular basis of abnormal calcium transients of failing human ventricular myocytes. Circ Res 2003; 92: 651-658.

[10] Priebe L and Beuckelmann DJ. Simulation study of cellular electric properties in heart failure. Circ Res 1998; 82: 1206-1223.

[11] Hunter PJ, McCulloch AD, and ter Keurs HEDJ. Modelling the mechanical properties of cardiac muscle. Prog Biophys Mol Biol 1998; 69: 289-331.

[12] Solovyova O, et al. Mechanical interaction of heterogeneous cardiac muscle segments in Silico: Effects on $\mathrm{Ca} 2+$ handling and action potential. Int $\mathrm{J}$ Bifurcation and Chaos 2003; 13: 3757-3782.

[13] Zicha S, et al. Transmural expression of transient outward potassium current subunits in normal and failing canine and human hearts. J Physiol 2004; 561(3): 735-748.

[14] Yan GX and Antzelevitch C. Cellular basis for the normal $\mathrm{T}$ wave and the electrocardiographic manifestations of the long-QT syndrome. Circulation 1998; 98:1928-1936.

[15] Hund TJ and Rudy Y. Rate dependence and regulation of action potential and calcium transient in a canine cardiac ventricular cell model. Circulation 2004; 110: 3168-3174.

[16] Akar FG and Rosenbaum DS. Transmural electrophysiological heterogeneities underlying arrhythmogenesis in heart failure. Circ Res 2003; 93: 638-645.

[17] Markhasin VS, et al. Mechano-electric interactions in heterogeneous myocardium: development of fundamental experimental and theoretical models. Prog Biophys Mol Biol 2003; 82: 207-220.

[18] Antzelevitch C and Fish J. Electrical heterogeneity within the ventricular wall. Basic Res Cardiol 2001; 96: 517-527.

Address for correspondence

Ling Xia

Department of Biomedical Engineering

Zhejiang University

Hangzhou 310027, China

E-mail: xialing@hzcnc.com 